\title{
Comprehensive Assessment of Left Atrial and Ventricular Remodeling in Paroxysmal Atrial Fibrillation by Cardiovascular Magnetic Resonance Myocardial Extracellular Volume Fraction and Feature Tracking Strain
}

Akimasa Yamada Nihon University

Naoki Hashimoto Nihon University

Hidesato Fujito

Nihon University

Takumi Hatta

Nihon University

Yuki Saito

Nihon University

Naoto Otsuka

Nihon University

Yuji Wakamatsu

Nihon University

Masaru Arai

Nihon University

Ryuta Watanabe

Nihon University

Sayaka Kurokawa

Nihon University

Daisuke Kitano

Nihon University

Koichi Nagashima

Nihon University

Shunichi Yoda

Nihon University

Yasuo Okumura ( $\nabla$ okumura.yasuo@nihon-u.ac.jp)

Nihon University 


\section{Research Article}

Keywords: paroxysmal atrial fibrillation, myocardial fibrosis, extracellular volume fraction, left atrial strain, LA/LV remodeling, CMR

Posted Date: February 12th, 2021

DOl: https://doi.org/10.21203/rs.3.rs-181256/v1

License: (c) (i) This work is licensed under a Creative Commons Attribution 4.0 International License.

Read Full License 


\section{Abstract}

Atrial fibrillation (AF) is a progressive disease that starts with structural or functional changes in the left atrium and left ventricle, and evolves from paroxysmal toward sustained forms. Early detection of structural or functional changes in the left atrium and left ventricle in the paroxysmal stage could be useful for identifying higher risk of progression to persistent AF and future cardio-cerebrovascular events. The aim of this study was to test the hypothesis that feature tracking (FT) left atrial (LA) strain and left ventricular (LV) extracellular volume fraction (ECV) derived from cardiovascular magnetic resonance (CMR) could detect the early changes of remodeling in the left atrium and ventricle in the stage of paroxysmal AF (PAF).Participants comprised 106 PAF patients (age, $66.1 \pm 10.7$ years; $66 \%$ male) who underwent clinical CMR before pulmonary vein isolation and 20 control subjects (age, $68.3 \pm 8.6$ years; $55 \%$ male). CMR-FT LA strain/phasic function and LV-ECV were compared between the PAF and control groups. Total and passive LA empty fraction (LAEF) and LA strain (corresponding to LA reservoir and conduit function) were decreased in the PAF group compared to the control group. However, active LAEF (corresponding to LA booster pump function) did not differ significantly between the PAF group (33.9 \pm $10.9 \%)$ and control group $(37.9 \pm 13.3 \%, p=0.15)$, while active LA strain (corresponding to LA booster pump function) was significantly decreased in the PAF group (11.4 \pm 4.3 vs. $15.2 \pm 5.6 \%, p=0.002)$. LVECV was significantly greater in the PAF group $(28.7 \pm 2.8 \%)$ than in the control group $(26.6 \pm 2.0 \%, p=$ $0.002)$. In the PAF group, LV-ECV correlated significantly with E/e' and LA volume index. Regarding LA strain, correlations were seen between LV-ECV and both reservoir function and conduit function.CMR-FT LA strain in combination with LV-ECV offers a potential imaging marker that identifies LA/LV remodeling including subtle LA booster pump dysfunction undetectable by conventional booster pump LAEF in the stage of PAF. These findings provide new insights into LA-LV interactions and further investigation regarding association between perpetuation of $A F$ and LA functional remodeling in PAF patients.

\section{Introduction}

Atrial fibrillation (AF) is the most common clinical arrhythmia, with an increasing prevalence worldwide, and contributes to morbidity and mortality. ${ }^{1,2}$ Our understanding of left atrial (LA) structure and function has advanced significantly over the past decade with the marked development of multimodality imaging. ${ }^{3}$ Accurate evaluation of LA structural and functional remodeling is potentially key to the optimal management of AF in clinical practice.

Cardiovascular magnetic resonance (CMR) is accurate, reproducible, and widely regarded as the noninvasive reference standard for structural, functional and tissue-characterizing assessments of the atrium and ventricle. ${ }^{4,5} \mathrm{CMR}$ can determine the extracellular volume fraction (ECV) of left ventricular (LV) myocardium, a hallmark of myocardial fibrosis, by measuring T1 relaxation times before and after administration of gadolinium contrast. ${ }^{6}$ An excellent correlation between myocardial ECV derived from CMR and quantitative histopathology has been confirmed in a number of studies. ${ }^{7,8}$ Feature tracking (FT) represents a novel method to quantify myocardial strain directly from standard cine images without the 
need for complex and time-consuming sequences. ${ }^{9}$ With the advent of FT technology, myocardial strain can now be assessed from routine CMR studies, overcoming several inherent limitations of speckletracking echocardiography. ${ }^{10}$ This novel approach has also recently been applied to assess atrial strain with its high spatial resolution and excellent endocardial border detection. ${ }^{11-14}$ Using these advanced approaches, CMR could have the potential to quantify LA strain and LV-ECV in a single study.

AF usually presents in a self-terminating paroxysmal form, over time, the type of AF often progresses to become more sustained and LA remodeling concomitantly develops. ${ }^{15}$ LA strain is an important imaging marker that correlates with functional LA remodeling and the risk of cardio-cerebrovascular events. ${ }^{16} \mathrm{~A}$ large body of literature has been published on the relationship between progression of LA remodeling and persistent $A F .{ }^{17-19}$ Despite intensive investigation in persistent $A F$, few studies have explored the characteristics of LA remodeling and LV myocardial fibrosis, particularly in the stage of paroxysmal AF (PAF). The present study therefore aimed to clarify whether feature tracking LA strain and LV-ECV derived from CMR could detect the early changes of remodeling in the left atrium and ventricle in the stage of PAF.

\section{Methods}

\section{Study population}

We conducted a prospective, single-center, observational study. Consecutive patients with PAF who were scheduled for pulmonary vein isolation (PVI) at our institution between July 2018 and September 2020 were recruited for this study. PAF was defined as AF that spontaneously terminates within 7 days. Inclusion criteria for the PAF group were: 1) patients scheduled for first PVI; 2) patients in sinus rhythm at the time of attendance at the outpatient clinic. Exclusion criteria were as follows: 1) severe renal failure (glomerular filtration rate $<30 \mathrm{~mL} / \mathrm{m}^{2}$ ); 2) severe valvular heart disease (e.g., severe aortic stenosis and regurgitation, and severe mitral regurgitation); 3) cardiomyopathy (e.g., hypertrophic cardiomyopathy, dilated cardiomyopathy, cardiac amyloidosis, cardiac sarcoidosis or iron overload); 4) myocardial infarction (by either history according to the presence of pathological Q waves on electrocardiography or wall motion abnormality on echocardiography). Patients were referred for CMR to image the pulmonary vein and screen for cardiac diseases. Twenty age- and sex-matched subjects with no history of cardiovascular disease and with normal results from physical examination, electrocardiography and echocardiography were recruited as healthy control subjects. This study was conducted in accordance with the principles of the Declaration of Helsinki and with the approval of the Institutional Review Board (Nihon University Itabashi Hospital Clinical Research Judging Committee:approval number RK-18041002). Written informed consent for study participation was obtained from each patient.

\section{CMR protocol}

MR images were acquired using a 1.5-T scanner (Ingenia; Philips Healthcare, Eindhoven, the Netherlands) with retrospective electrocardiographic gating. Sequences were acquired during breath-holds in the 
supine position. The comprehensive CMR protocol consisted of standard steady-state free precession (SSFP) cine MRI, T1 mapping by a modified look-Locker inversion recovery sequence (MOLLI) before contrast, late gadolinium enhancement (LGE) MRI, and T1 mapping by MOLLI after contrast. Standard SSFP cine images included coverage of the entire LV and LA using short-axis slices and 2-, 3-, and 4chamber views with temporal resolution $<40 \mathrm{~ms}$. LGE imaging was acquired with a T1-weighted inversion recovery gradient-echo sequence $15 \mathrm{~min}$ after contrast administration $(0.15 \mathrm{mmol} / \mathrm{kg}, \mathrm{Gd}-\mathrm{BTD}$ 03A, Gadovist; Bayer Japan, Tokyo, Japan) in three long-axis slices (two-, three-, and four-chamber) and a stack of short-axis slices completely encompassing the LV. To calculate ECV, T1 measurements were acquired in a single breath-hold MOLLI sequence in three short-axis (basal, mid-ventricular, and apex) and two long-axis slices before and 20 min after contrast administration. Detailed CMR sequence parameters are available as supplemental materials.

\section{Image analysis}

All images were analyzed by a blinded observer using commercial post-processing software (Circle CVI 42; Circle Cardiovascular Imaging Inc., Calgary, Canada).

\section{Volumetric and functional analyses}

Endo- and epicardial LV contours were manually drawn in short-axis cine images covering from the mitral valve to the apex at end-diastole and end-systole to calculate end-diastolic and end-systolic volume, stroke volume, and ejection fraction. LV mass was calculated as the sum of myocardial volumes multiplied by the specific gravity $(1.05 \mathrm{~g} / \mathrm{mL})$ of myocardial tissue. Papillary muscles were excluded from LV mass. LA volumes (LAV) were calculated using the biplane area-length method as previously described. ${ }^{20} \mathrm{LAV}$ were assessed at end-systole, just before opening of the mitral valve $\left(\mathrm{LAV}_{\text {max }}\right)$, at enddiastole just before closure of the mitral valve $\left(\mathrm{LAV}_{\mathrm{min}}\right)$ and at diastole just before $L A$ contraction $\left(\mathrm{LAV}_{\text {pre-ac }}\right)$. In addition, LA total, passive, and active emptying fraction (EF) were calculated with the following formulas: ${ }^{21}$

total LAEF $=\left(\mathrm{LAV}_{\max }-\mathrm{LAV}_{\min }\right) / \mathrm{LAV}_{\max } \times 100$

passive $L A E F=\left(L_{\text {max }}-L_{\text {m }} V_{\text {pre-ac }}\right) / L A V_{\max } \times 100$

active $L A E F=\left(L A V_{\text {pre-ac }}-L A V_{\text {min }}\right) / L A V_{\text {pre-ac }} \times 100$

Feature tracking strain analysis

\section{Left ventricular strain}

Endo- and epicardial borders were semi-automatically drawn at end-diastole in short- and long-axis cines, excluding papillary muscles from the endocardial contour, then automatically propagated to all slices throughout the cardiac cycle. Tracking was visually reviewed and manually corrected in case of inaccurate automated border tracking. Short-axis cines were tracked to derive radial and circumferential strain, while 2-, 3-, and 4-chamber-view cines were tracked to derive longitudinal strain. 


\section{Left atrial strain}

At end diastole, endo- and epicardial borders were manually traced in 4- and 2-chamber views before the automated tracking algorithm was applied (pulmonary veins and LA appendage were excluded). Tracing was blindly repeated three times in 4- and 2-chamber views, and the results of LA strain and strain rate (SR) from the three repetitions were averaged in both views. As previously described, ${ }^{11}$ three aspects of atrial strain were derived: passive strain (corresponding to atrial conduit function), active strain (corresponding to atrial booster pump function) and total strain (corresponding to atrial reservoir function). Accordingly, three SR parameters were also calculated: peak early negative strain rate (SRp, corresponding to atrial conduit function), peak late negative strain rate (SRa, corresponding to atrial booster pump function) and peak positive strain rate (SRt, corresponding to atrial reservoir function) (Fig. 1).

\section{LV-ECV calculation}

To calculate LV-ECV, native T1 and post-contrast T1 values were measured by drawing LV endocardial and epicardial borders with care to avoid the blood pool contamination on a series of three short-axis views before and after contraction administration. LV-ECV was then calculated as follows: ${ }^{22}$ ECV $=(1-$ hematocrit $) \times\left\{\left[\left(1 / T 1_{\text {myocardium.post }}\right)-\left(1 / \mathrm{T} 1_{\text {myocardium.pre }}\right)\right] /\left[\left(1 / \mathrm{T} 1_{\text {blood.post }}\right)-\left(1 / \mathrm{T} 1_{\text {blood.pre }}\right)\right]\right\} \times 100$.

\section{Statistical analysis}

Normality was assessed using the Shapiro-Wilk test. All data are shown as mean \pm standard division, median (interquartile range), or number of participants (percentages), as appreciate.

Comparison of the continuous variables between the two groups was performed by independent $t$ tests and Mann-Whitney $U$ tests. Categorical variables were assessed using the chi-square test or Fisher's exact test, as appropriate. Correlations between each group between continuous indices were assessed using Pearson's and Spearman's correlation coefficients for parametric and nonparametric data, respectively. A blinded list of mixed PAF patients and control subjects in random order was used for image analysis. Two independent observers measured LV-ECV and LA strain in 20 randomly selected subjects (10 PAF and 10 control subjects) to assess interobserver reproducibility. Furthermore, one observer measured LVECV and LA strain twice with a washout period of 3 months to determine intraobserver reproducibility. The inter- and intraobserver reproducibility of the LV-ECV measurement and LA strain were tested by calculating interclass correlation coefficient (ICC). Values of $\mathrm{P}<0.05$ were considered significant. Statistical analyses were performed using SPSS version 23 software (SPSS, Chicago, IL).

\section{Results}

\section{Baseline patient characteristics}


Of a total of 129 patients with PAF, 23 patients who had severe artifacts due to body or respiratory motions $(n=4), \operatorname{LGE}(n=5)$, or AF rhythm at the time of study $(n=14)$ were excluded. The remaining 106 PAF patients were analyzed for this study. Echocardiography was performed in all patients within a week from CMR examination. Demographic data for 106 PAF patients and 20 control subjects are summarized in Table 1. No significant differences in age, sex, heart rate, hematocrit (at the day of CMR examination), medications, lifestyle disease, $\mathrm{CHADS}_{2}$ score, or $\mathrm{CHA}_{2} \mathrm{DS}_{2}$-VASc score except BMI were apparent between the groups. Usages of both anticoagulant and antiarrhythmic drugs were more frequent in patients with PAF $(P<0.0001$, respectively). 
Table 1

Demographic data of the PAF and control groups.

\begin{tabular}{|c|c|c|c|}
\hline & $\begin{array}{l}\text { PAF } \\
(n=106)\end{array}$ & $\begin{array}{l}\text { Control } \\
(n=20)\end{array}$ & $P$ value \\
\hline Age (years) & $66.1 \pm 10.7$ & $68.3 \pm 8.6$ & 0.50 \\
\hline Male $(n, \%)$ & $70(66 \%)$ & $11(55 \%)$ & 0.35 \\
\hline $\operatorname{BMI}\left(\mathrm{kg} / \mathrm{m}^{2}\right)$ * & $24.3 \pm 3.7$ & $22.3 \pm 2.9$ & 0.04 \\
\hline $\mathrm{SBP}(\mathrm{mmHg})$ & $132.7 \pm 17.7$ & $132.0 \pm 18.9$ & 0.87 \\
\hline $\mathrm{DBP}(\mathrm{mmHg})$ & $77.2 \pm 13.8$ & $76.6 \pm 10.2$ & 0.86 \\
\hline HR (beats/min) & $72.0 \pm 14.7$ & $71.6 \pm 9.4$ & 0.78 \\
\hline Hematocrit (\%) & $40.6 \pm 4.4$ & $41.5 \pm 3.6$ & 0.38 \\
\hline $\operatorname{eGFR}\left(\mathrm{mL} / \mathrm{min} / 1.73 \mathrm{~m}^{2}\right)$ & $73.0 \pm 14.2$ & $74.1 \pm 22.8$ & 0.27 \\
\hline NT-proBNP (pg/mL) & $209.4 \pm 333.5$ & $289.2 \pm 731.0$ & 0.34 \\
\hline Medical and drug history & $9(3-12)$ & - & \\
\hline Hypertension (n, \%) & $64(60 \%)$ & $13(65 \%)$ & 0.70 \\
\hline Diabetes (n, \%) & $17(16 \%)$ & $5(25 \%)$ & 0.33 \\
\hline Dyslipidemia (n, \%) & $38(36 \%)$ & $10(50 \%)$ & 0.23 \\
\hline $\operatorname{CAD}(n, \%)$ & $1(1 \%)$ & $1(5 \%)$ & 0.18 \\
\hline Heart failure (n, \%) & $7(7 \%)$ & $1(5 \%)$ & 0.79 \\
\hline Stroke $(n, \%)$ & $12(11 \%)$ & $1(5 \%)$ & 0.39 \\
\hline $\mathrm{CHADS}_{2}$ score & $1.3 \pm 1.1$ & $1.2 \pm 0.8$ & 0.76 \\
\hline $\mathrm{CHA}_{2} \mathrm{DS}_{2}$-VASc score & $2.2 \pm 1.4$ & $2.4 \pm 1.4$ & 0.50 \\
\hline Duration of PAF (month) & $9(3-12)$ & - & - \\
\hline ACEi / ARB $(n, \%)$ & $37(35 \%)$ & $7(35 \%)$ & 0.99 \\
\hline Calcium channel blocker $(\mathrm{n}, \%)$ & $39(37 \%)$ & $8(40 \%)$ & 0.79 \\
\hline
\end{tabular}

All data are shown as mean \pm standard division, median (interquartile range), or number of participants (percentages), as appreciate.

ACEi: angiotensin-converting enzyme inhibitor; ARB: angiotensin II receptor blocker; BMI: body mass index; CAD: coronary artery disease; DBP: diastolic blood pressure; eGFR: estimated glomerular filtration rate; HR: heart rate; NT-proBNP: $\mathrm{N}$ terminal pro B type natriuretic peptide; PAF: paroxysmal atrial fibrillation; SBP: systolic blood pressure. ${ }^{*} P<0.05$. 


\begin{tabular}{|c|c|c|c|}
\hline & $\begin{array}{l}\text { PAF } \\
(n=106)\end{array}$ & $\begin{array}{l}\text { Control } \\
(n=20)\end{array}$ & $P$ value \\
\hline Beta-blocker (n, \%) & $50(47 \%)$ & $5(25 \%)$ & 0.07 \\
\hline Diuretic (n, \%) & $8(8 \%)$ & $1(5 \%)$ & 0.67 \\
\hline Statin $(n, \%)$ & $33(31 \%)$ & $6(30 \%)$ & 0.92 \\
\hline Antidiabetic drugs / Insulin (n, \%) & $12(11 \%)$ & $0(0 \%)$ & 0.11 \\
\hline Anticoagulant drugs $(\mathrm{n}, \%)$ * & $106(100 \%)$ & $2(10 \%)$ & $<0.0001$ \\
\hline Number of antiarrhythmic drugs * & $1.3 \pm 0.9$ & $0.2 \pm 0.4$ & $<0.0001$ \\
\hline \multicolumn{4}{|c|}{$\begin{array}{l}\text { All data are shown as mean } \pm \text { standard division, median (interquartile range), or number of } \\
\text { participants (percentages), as appreciate. }\end{array}$} \\
\hline \multicolumn{4}{|c|}{$\begin{array}{l}\text { ACEi: angiotensin-converting enzyme inhibitor; ARB: angiotensin II receptor blocker; BMI: body mass } \\
\text { index; CAD: coronary artery disease; DBP: diastolic blood pressure; eGFR: estimated glomerular } \\
\text { filtration rate; HR: heart rate; NT-proBNP: N terminal pro B type natriuretic peptide; PAF: paroxysmal } \\
\text { atrial fibrillation; SBP: systolic blood pressure. }{ }^{\star} P<0.05 \text {. }\end{array}$} \\
\hline
\end{tabular}

\section{LV function and strain}

The results of LV function and strain between the groups are shown in Table 2. No significant differences were seen in LV volumetric or functional parameters. Global radial strain, global circumferential strain, and global longitudinal strain did not differ significantly between the groups. 
Table 2

Comparison of left ventricular function and strain between the PAF and control groups.
$\operatorname{PAF}(n=106)$
Control $(n=20)$
$P$ value

LV function

$\operatorname{LVEF}(\%)$

$61.1 \pm 4.8$

$62.2 \pm 4.0$

0.35

$\operatorname{LVEDVI}\left(\mathrm{mL} / \mathrm{m}^{2}\right)$

$74.1 \pm 14.5$

$74.8 \pm 12.5$

0.84

LVESVI $\left(\mathrm{mL} / \mathrm{m}^{2}\right)$

$29.0 \pm 7.4$

$28.6 \pm 7.3$

0.60

LVSVI $\left(\mathrm{mL} / \mathrm{m}^{2}\right)$

$45.2 \pm 8.9$

$46.3 \pm 6.3$

0.61

LVMI $\left(\mathrm{g} / \mathrm{m}^{2}\right)$

$44.2 \pm 8.4$

$45.9 \pm 6.9$

0.30

\section{LV strain (\%)}

\begin{tabular}{llll|} 
GRS & $29.6 \pm 8.1$ & $32.9 \pm 9.6$ & 0.27 \\
GCS & $-17.3 \pm 3.2$ & $-18.3 \pm 3.3$ & 0.22 \\
\hline GLS & $-12.7 \pm 2.3$ & $-13.2 \pm 2.5$ & 0.35
\end{tabular}

All data are shown as mean \pm standard deviation.

GCS: global circumferential strain; GLS: global longitudinal strain; GRS: global radial strain; LVEDVI: left ventricular end diastolic volume index; LVEF: left ventricular ejection fraction; LVESVI: left ventricular end systolic volume index; LVMI: left ventricular mass index; LVSVI: left ventricular stroke volume index.

\section{LA phasic function and strain}

The results of LA phasic function and strain are summarized in Table 3. Representative cases of a patient with PAF and a control subject are shown in Fig. 2. LAVI $\left(\mathrm{V}_{\text {max }}, \mathrm{V}_{\text {pre-ac }}, \mathrm{V}_{\text {min }}\right)$ was significantly greater in the PAF group than in the control group (LAVI $\mathrm{Lax}_{\text {: }}: 43.6 \pm 17.6$ vs. $35.6 \pm 18.0 \mathrm{~mL} / \mathrm{m}^{2}, P=0.01$; $\mathrm{LAVI}_{\text {pre-ac: }}$ : $34.6 \pm 15.4$ vs. $26.1 \pm 16.4 \mathrm{~mL} / \mathrm{m}^{2}, P=0.002 ; \mathrm{LAVI}_{\min }: 23.9 \pm 14.5$ vs. $\left.17.6 \pm 16.9 \mathrm{~mL} / \mathrm{m}^{2}, P=0.004\right)$. Total and passive LAEF and LA strain (corresponding to reservoir and conduit functions) were significantly decreased in the PAF group than in the control group (total LAEF: $48.0 \pm 10.7$ vs. $55.4 \pm 13.1 \%, P=0.004$; passive LAEF: $21.5 \pm 7.3$ vs. $29.0 \pm 7.5 \%, P<0.0001$; total LA strain: $22.3 \pm 7.6$ vs. $29.3 \pm 10.2 \%, P<0.0001$; passive LA strain: $10.9 \pm 4.6$ vs. $14.1 \pm 5.8 \%, P=0.002$ ). Active LAEF (corresponding to booster pump function) did not differ between the PAF and control groups ( $33.9 \pm 10.9 \%$ vs. $37.9 \pm 13.3 \%, P=0.15)$, while active LA strain was significantly decreased in the PAF group $(11.4 \pm 4.3$ vs. $15.2 \pm 5.6 \%, P=0.002)$. 
Table 3

Comparison of left atrial function and strain between the PAF and control groups.

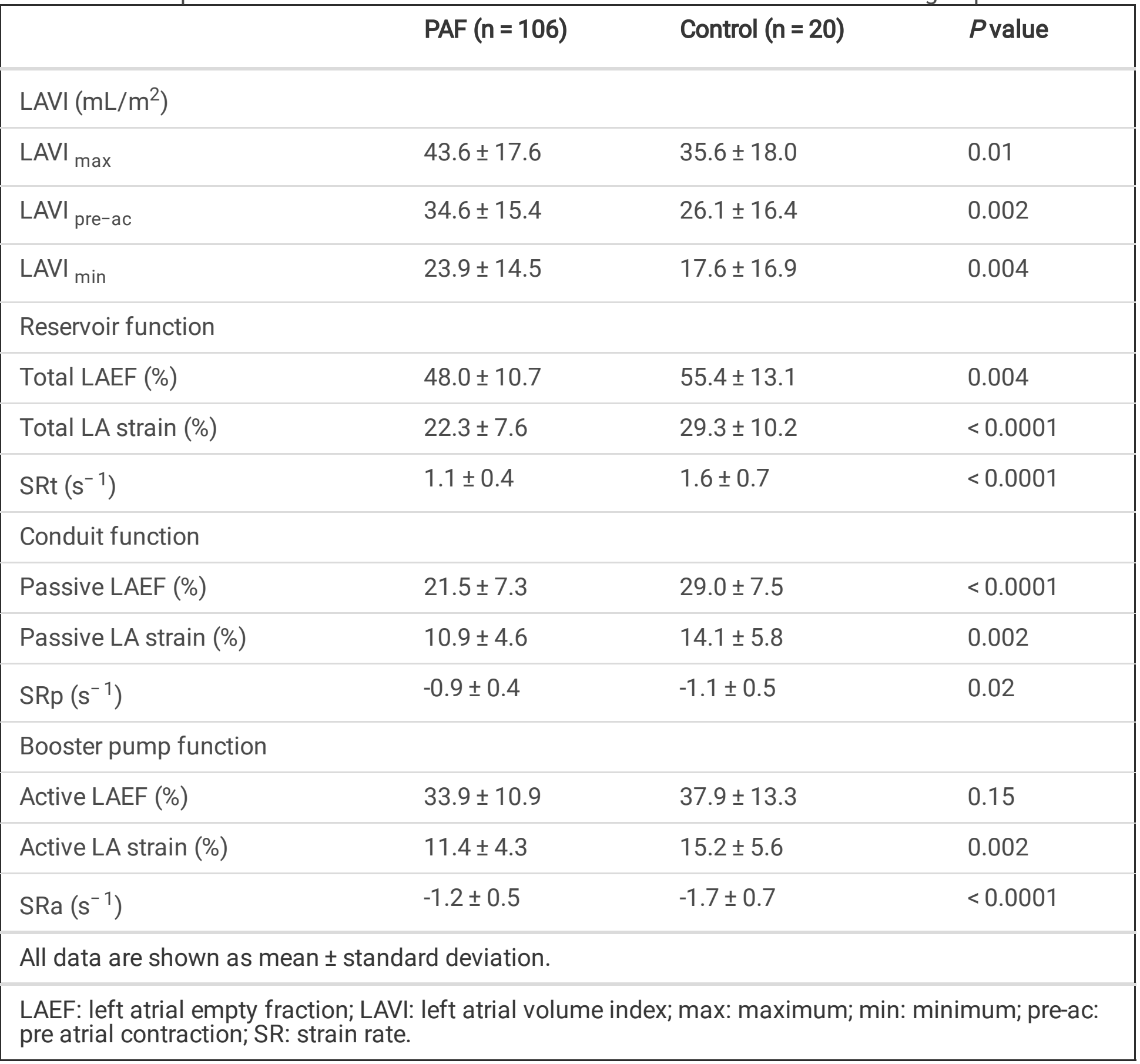

\section{LV-ECV}

LV-ECV was significantly greater in the PAF group $(28.7 \pm 2.8 \%)$ than in the control group $(26.6 \pm 2.0 \%, P=$ 0.002) (Fig. 3).

\section{Correlation between LV-ECV and cardiac parameters in PAF patients}

Correlations between LV-ECV and various parameters in PAF patients are summarized in Table 4. In the PAF group, LV-ECV correlated significantly with NT-proBNP $(r=0.33, P=0.001)$, E/e' $(r=0.30, P=0.002)$, 
and LAVI (LAV $\max _{\text {ax }}: r=0.31, P=0.001$, LAV pre-ac $: r=0.32, P=0.001$; $\left.\operatorname{LAV}_{\text {min }}: r=0.30, P=0.002\right)$, but not with LV mass index $(r=-0.08, P=0.42)$ or $\operatorname{LVEF}(r=-0.02, P=0.85)$. Regarding LA strain, correlations were apparent between LV-ECV and LA reservoir function (total LA strain: $r=-0.21, P=0.03$ ) and LA conduit function (passive LA strain: $r=-0.25, P=0.009$ ), while no correlation with LA booster pump function (active LA strain: $r=-0.09, P=0.38$ ) was evident in PAF patients. 
Correlation of LV-ECV in PAF patients.

\section{LV-ECV}

$\begin{array}{lll}\text { Parameters } & r & P \text { value } \\ \text { Age } & 0.18 & 0.06 \\ \text { NT-proBNP * } & 0.33 & 0.001 \\ \text { LVEF } & -0.019 & 0.85 \\ \text { LVMI } & -0.08 & 0.42 \\ \text { E/e' * } & 0.30 & 0.002 \\ \text { LAVI }_{\text {max }} \text { * } & 0.31 & 0.001 \\ \text { LAVI }_{\text {pre-ac }} \text { * } & 0.32 & 0.001 \\ \text { LAVI }_{\text {min }} \text { * } & 0.30 & 0.002\end{array}$

Reservoir function

\begin{tabular}{lll} 
Total LAEF $(\%) *$ & -0.19 & 0.05 \\
\hline Total LA strain $(\%) *$ & -0.21 & 0.03 \\
SRt $\left(\mathrm{s}^{-1}\right)$ & -0.17 & 0.08
\end{tabular}

Conduit function

\begin{tabular}{lll} 
Passive LAEF (\%) & -0.18 & 0.07 \\
\hline Passive LA strain $(\%) *$ & -0.25 & 0.009 \\
\hline SRp $\left(s^{-1}\right)$ * & 0.28 & 0.004
\end{tabular}

Booster pump function

$\begin{array}{lll}\text { Active LAEF }(\%) & -0.14 & 0.15 \\ \text { Active LA strain (\%) } & -0.09 & 0.38 \\ \text { SRa }\left(\mathrm{s}^{-1}\right) & 0.14 & 0.16\end{array}$

ECV: extracellular volume fraction; LAEF: left atrial empty fraction; LAVI: left atrial volume index; LV: left ventricular; LVEF: left ventricular ejection fraction; LVMI: left ventricular mass index; NT-proBNP: N terminal pro B type natriuretic peptide; SR: strain rate. ${ }^{\star} P<0.05$.

\section{Intra- and interobserver reproducibility}

ICC for the intra- and interobserver measurements of LV-ECV showed almost-perfect agreement. All LA strain and SR parameters displayed good to excellent reproducibility for both intra- and interobserver 
classes (Table 5).

Table 5

Intraobserver and interobserver reproducibility for LV-ECV and LA strain parameters.

$$
\text { ICC (95\% Cl) }
$$

\section{Intraobserver}

LV-ECV

LA total strain

LA passive strain

LA active strain

LA SRt

LA SRp

LA SRa
$0.98(0.96-0.99)$

$0.88(0.69-0.95)$

0.89 (0.71-0.96)

$0.88(0.69-0.95)$

$0.76(0.40-0.91)$

$0.89(0.73-0.96)$

$0.86(0.64-0.94)$
Interobserver

$0.98(0.95-0.98)$

$0.86(0.65-0.95)$

$0.78(0.45-0.91)$

$0.80(0.49-0.92)$

$0.73(0.32-0.89)$

$0.81(0.52-0.93)$

$0.79(0.47-0.92)$

ECV: extracellular volume fraction; ICC: intraclass correlation coefficient; LA: left atrial; LV: left ventricular; SRa: peak late negative strain rate; SRp: peak early negative strain rate; SRt: peak positive strain rate.

\section{Discussion}

This study disclosed the difference in the extent of LV myocardial fibrosis and LA functional remodeling by the simultaneous assessment of LV-ECV and feature tracking LA strain derived from a single CMR study between PAF patients and control subjects. The main findings of this study were: 1) CMR-FT LA strain could identify subtle LA booster pump dysfunction undetectable by conventional active LAEF; 2 ) LV-ECV was significantly greater in PAF patients than in control subjects, indicating advanced LV myocardial fibrosis; 3) significant correlations were evident between LV-ECV and LA reservoir function and LA conduit function in the PAF group; 4) LV-ECV and LA strain parameters displayed robust reproducibility for both intra- and interobserver classes.

\section{LA remodeling}

LA remodeling involves structural and functional changes, and those changes in LA already coexist before the development and persistence of $A F^{23}$ Early detection of these changes in individuals is helpful for optimal management of $A F$ in clinical practice. LA dilation reflected by LA structural remodeling is a well-known risk for AF development and stroke. ${ }^{24,25}$ As expected, our study showed LA volumes were significantly higher in the PAF group than in the control group. Nonetheless, in our cohort, the percentage of LA dilation in PAF was relatively low $(26 \%)$ and mean $\operatorname{LAV}_{\max }\left(43.6 \pm 17.6 \mathrm{~mL} / \mathrm{m}^{2}\right)$ was within the normal range $\left(26-52 \mathrm{~mL} / \mathrm{m}^{2}\right) .{ }^{26}$ Given the complex atrial geometry and thin atrial wall thickness, cardiovascular imaging for noninvasive assessment of LA function is challenging. With the remarkable 
developments in CMR imaging (excellent spatial resolution to identify heterogeneous atrial wall thickness), complex LA function can now be accurately assessed..$^{12}$ LA function has been divided into three components: ${ }^{21}$ reservoir function when the atria store blood in systole, as a conduit when blood flows passively into the LV in early diastole, and as a booster pump when the atria contract in late diastole. In our study, total and passive LAEF (corresponding to reservoir and conduit functions) were decreased in the PAF group as compared to the control group, in line with a previous report. ${ }^{23}$ However, our study demonstrated that active LAEF (corresponding to booster pump function) did not differ significantly between the PAF and control groups. Whether active LAEF is reduced ${ }^{23}$ or not $^{27}$ in PAF patients has remained controversial. In an animal experiment, AF itself induced booster pump dysfunction by causing a tachycardia-induced atrial cardiomyopathy. ${ }^{28}$ Shin et al. demonstrated that active LAEF was significantly reduced in patients with frequent episodes of AF than in others. ${ }^{29}$ Alternatively, even in patients with PAF, booster pump function tends to be unaffected when the basic cardiac rhythm is almost sinus rhythm. A potential explanation for similar active LAEF between the PAF and control groups in our study was that the frequency of PAF episodes had been low and the duration of PAF had been short, since we selected PAF patients presenting with sinus rhythm at both outpatient clinic and CMR examinations. LA remodeling in the PAF group might thus be mild and booster pump function assessed by LAEF was preserved in the present study.

Myocardial strain is more sensitive imaging marker to detect early changes of cardiac function than EF, as is the case with incipient disease such as PAF. ${ }^{30}$ We found that CMR-FT LA strain could identify subtle LA booster pump dysfunction undetectable by conventional active LAEF. A recent study showed LA strain to be the strongest independent predictor of progression to persistent $A F$ in a model including $L A$ diameter, volume, and function. ${ }^{31}$ In this context, a large registry demonstrated that LA strain and p-waveto-A' duration on echocardiographic tissue Doppler imaging was independently associated with stroke risk in a model including $\mathrm{CHA}_{2} \mathrm{DS}_{2}$-VASc score, age, and anticoagulant use. ${ }^{32}$ Moreover, emerging data suggest an independent, inverse association between LA strain measured using CMR and incident heart failure. ${ }^{33}$ These findings suggested that early detection of LA dysfunction by CMR-FT LA strain in the stage of PAF could help to identify patients at high risk of progression to persistent AF and future cardiocerebrovascular events.

\section{LV remodeling}

The pathogenesis of LV fibrosis in PAF has not been fully elucidated. LV fibrosis may occur secondary to AF as a consequence of rapid ventricular rates or the irregularity of ventricular contraction. ${ }^{34,35}$ Alternatively, PAF may occur secondary to diastolic dysfunction caused by LV fibrosis as a consequence of primary cardiomyopathy or conditions such as hypertension, diabetes mellitus, obesity, and aging. ${ }^{36,37}$ A histological study by Frustaci et al. showed that LV endomyocardial biopsy in 14 lone AF patients demonstrated nonspecific necrosis or fibrosis in $60 \% .{ }^{38}$ We have previously demonstrated that ventricular fibrotic changes are more pronounced in AF patients than in subjects with sinus rhythm according to echocardiography-derived integrated backscatter. ${ }^{39}$ CMR-based myocardial ECV has been regarded as the 
most robust noninvasive measurement for quantifying myocardial fibrosis. ${ }^{7,8}$ However, evidence for the association between LV-ECV and AF remains limited. ${ }^{40}$ Moreover, direct comparisons regarding LV-ECV between PAF and control subjects have not been conducted previously. In this study, CMR-based LV-ECV revealed that myocardial fibrosis in the setting of PAF was more advanced than in control subjects. We also found a significant correlation between LV-ECV and LA volume index in PAF patients. Elevated LVECV is reported to be a major contributor to the impaired LV relaxation and stiffness. ${ }^{41}$ With increased LV stiffness, left atrial pressure rises to maintain adequate ventricular filling, and the increased atrial wall tension leads to subsequent left atrial enlargement. Furthermore, LV-ECV in PAF patients significantly correlated with LA reservoir function and LA conduit function, suggesting a potential link between LV remodeling and LA functional remodeling.

\section{Limitations}

This study has some limitations that need to be acknowledged when interpreting the results. First, LV-ECV and LA/LV strain data in our study were derived from a single center and a single vendor. A multicenter, multivendor study is warranted to validate the results of our study. Second, subjects in this study were ineligible for cardiac catheterization including endomyocardial biopsy and pressure study, and histological validation and physiological parameters regarding atrial and ventricular pressure were not obtained. However, a number of studies have shown myocardial ECV as determined by CMR correlated excellently with histological quantification of myocardial fibrosis and association between atrial pressure and atrial remodeling. ${ }^{42}$ Third, the association of LA strain and electrophysiological parameters was not analyzed. Moreover, the impact of PVI for PAF on LA/LV remodeling was also undetermined, and continued research is required regarding these points. Finally, this study did not address clinical outcomes such as progression to persistent AF or cardio-cerebrovascular events, and further studies are warranted to clarify the relationship between LA/LV remodeling and future cardio-cerebrovascular events.

\section{Conclusion}

CMR-FT LA strain in combination with LV-ECV offers a potential imaging marker that identifies LA/LV remodeling including subtle LA booster pump dysfunction undetectable by conventional booster pump LAEF in the stage of PAF. These findings provide new insights into LA-LV interactions and further investigation regarding association between perpetuation of $A F$ and $L A$ functional remodeling in PAF patients.

\section{Abbreviations}

$\mathrm{AF}$

Atrial fibrillation; BMl:body mass index; CMR:Cardiovascular magnetic resonance; ECV:Extracellular volume fraction; EF:Emptying fraction; FT:Feature tracking; ICC:interclass correlation coefficient; LA:Left atrial/atrium; LAV:Left atrial volume; LAVI:left atrial volume index; LGE:late gadolinium enhancement; LV:left ventricular; MOLLI:modified look-Locker inversion recovery sequence; PAF:paroxysmal atrial 
fibrillation; pre-ac:pre-atrial contraction; PVI:pulmonary vein isolation; SR:strain rate; SSFP:standard steady-state free precession

\section{Declarations}

\section{Acknowledgements}

Not applicable

\section{Authors' contributions}

AY participated in study design, conducted the statistical analysis, analyzed and interpreted the data, and drafted the manuscript. NH, HF, TH, YS analyzed the data. NO, YW, MA, RW, SK, DK, KN, SY recruited the subjects. OY revised the manuscript. The authors have read and approved the final manuscript.

\section{Funding}

This research did not receive any specific grant from funding agencies in the public, commercial, or notfor-profit sectors.

\section{Availability of data and materials}

The datasets used and/or analyzed during the current study available from the corresponding author on reasonable request.

\section{Ethics approval and consent to participate}

This study was conducted in accordance with the principles of the Declaration of Helsinki and with the approval of the Nihon University Institutional Review Board (approval number RK-180410-02, Aprl 24 ${ }^{\text {th }}$, 2018). All participants gave their written informed consent prior to participation.

\section{Consent for publication}

All data used or contained in this study were consented for publication as stipulated by Institutional Review Board policy.

\section{Competing interests}

The authors declare that they have no competing interests.

\section{Author details}

Department of Cardiovascular Medicine, Nihon University Graduate School

30-1 Oyaguchi kamimachi, Itabashi, Tokyo 173-8610, Japan 


\section{References}

1. Chugh, S. S. et al. Worldwide epidemiology of atrial fibrillation: a Global Burden of Disease 2010 Study. Circulation. 129, 837-847 (2014).

2. John, R. M., Michaud, G. F. \& Stevenson, W. G. Atrial fibrillation hospitalization, mortality, and therapy. Eur Heart J. 39, 3958-3960 (2018).

3. Donal, E., Galli, E., Lederlin, M., Martins, R. \& Schnell, F. Multimodality Imaging for Best Dealing With Patients in Atrial Arrhythmias. JACC Cardiovasc Imaging. 12, 2245-2261 (2019).

4. Bellenger, N. G. et al. Comparison of left ventricular ejection fraction and volumes in heart failure by echocardiography, radionuclide ventriculography and cardiovascular magnetic resonance; are they interchangeable? Eur Heart J. 21, 1387-1396 (2000).

5. Flett, A. S. et al. Equilibrium contrast cardiovascular magnetic resonance for the measurement of diffuse myocardial fibrosis: preliminary validation in humans. Circulation. 122, 138-144 (2010).

6. Taylor, A. J., Salerno, M., Dharmakumar, R. \& Jerosch-Herold, M. T1 Mapping: Basic Techniques and Clinical Applications. JACC Cardiovasc Imaging. 9, 67-81 (2016).

7. Kammerlander, A. A. et al. T1 Mapping by CMR Imaging: From Histological Validation to Clinical Implication. JACC Cardiovasc Imaging. 9, 14-23 (2016).

8. de Meester de Ravenstein, C. et al. Histological Validation of measurement of diffuse interstitial myocardial fibrosis by myocardial extravascular volume fraction from Modified Look-Locker imaging (MOLLI) T1 mapping at 3 T. J Cardiovasc Magn Reson. 17, 48 (2015).

9. Maret, E. et al. Functional measurements based on feature tracking of cine magnetic resonance images identify left ventricular segments with myocardial scar. Cardiovasc Ultrasound. 7, 53 (2009).

10. Muraru, D., Niero, A., Rodriguez-Zanella, H., Cherata, D. \& Badano, L. Three-dimensional speckletracking echocardiography: benefits and limitations of integrating myocardial mechanics with threedimensional imaging. Cardiovasc Diagn Ther. 8, 101-117 (2018).

11. Kowallick, J. T. et al. Quantification of left atrial strain and strain rate using Cardiovascular Magnetic Resonance myocardial feature tracking: a feasibility study. J Cardiovasc Magn Reson. 16, 60 (2014).

12. Zareian, M. et al. Left atrial structure and functional quantitation using cardiovascular magnetic resonance and multimodality tissue tracking: validation and reproducibility assessment. $J$ Cardiovasc Magn Reson. 17, 52 (2015).

13. Kowallick, J. T. et al. Quantification of atrial dynamics using cardiovascular magnetic resonance: inter-study reproducibility. J Cardiovasc Magn Reson. 17, 36 (2015).

14. Yang, Y. et al. Quantification of left atrial function in patients with non-obstructive hypertrophic cardiomyopathy by cardiovascular magnetic resonance feature tracking imaging: a feasibility and reproducibility study. J Cardiovasc Magn Reson. 22, 1 (2020).

15. de Vos, C. B. et al. Progression from paroxysmal to persistent atrial fibrillation clinical correlates and prognosis. J Am Coll Cardiol. 55, 725-731 (2010). 
16. Inoue, Y. Y. et al. Quantitative tissue-tracking cardiac magnetic resonance (CMR) of left atrial deformation and the risk of stroke in patients with atrial fibrillation. J Am Heart Assoc. 4, (2015). e001844

17. Walters, T. E. et al. Progression of atrial remodeling in patients with high-burden atrial fibrillation: Implications for early ablative intervention. Heart Rhythm. 13, 331-339 (2016).

18. Mahnkopf, C. et al. Evaluation of the left atrial substrate in patients with lone atrial fibrillation using delayed-enhanced MRI: implications for disease progression and response to catheter ablation. Heart Rhythm. 7, 1475-1481 (2010).

19. Kallergis, E. M. et al. Extracellular matrix alterations in patients with paroxysmal and persistent atrial fibrillation: biochemical assessment of collagen type-I turnover. J Am Coll Cardiol. 52, 211-215 (2008).

20. Sievers, B. et al. Assessment of left atrial volumes in sinus rhythm and atrial fibrillation using the biplane area-length method and cardiovascular magnetic resonance imaging with TrueFISP. $J$ Cardiovasc Magn Reson. 6, 855-863 (2004).

21. Järvinen, V., Kupari, M., Hekali, P. \& Poutanen, V. P. Assessment of left atrial volumes and phasic function using cine magnetic resonance imaging in normal subjects. Am J Cardiol. 73, 1135-1138 (1994).

22. Kehr, E., Sono, M., Chugh, S. S. \& Jerosch-Herold, M. Gadolinium-enhanced magnetic resonance imaging for detection and quantification of fibrosis in human myocardium in vitro. Int $J$ Cardiovasc Imaging. 24, 61-68 (2008).

23. Kojima, T. et al. Left atrial global and regional function in patients with paroxysmal atrial fibrillation has already been impaired before enlargement of left atrium: velocity vector imaging echocardiography study. Eur Heart J Cardiovasc Imaging. 13, 227-234 (2012).

24. Habibi, M. et al. Cardiac Magnetic Resonance-Measured Left Atrial Volume and Function and Incident Atrial Fibrillation: Results From MESA (Multi-Ethnic Study of Atherosclerosis). Circ Cardiovasc Imaging. 9, (2016). 10.1161/CIRCIMAGING.115.004299 e004299

25. Di Tullio, M. R., Sacco, R. L., Sciacca, R. R. \& Homma, S. Left atrial size and the risk of ischemic stroke in an ethnically mixed population. Stroke. 30, 2019-2024 (1999).

26. Kawel-Boehm, N. et al. Normal values for cardiovascular magnetic resonance in adults and children. J Cardiovasc Magn Reson. 17, 29 (2015).

27. Hong, J. et al. Left atrial functional remodeling in lone atrial fibrillation: a two-dimensional speckle tracking echocardiographic study. Echocardiography. 30, 1051-1060 (2013).

28. Sun, H., Gaspo, R., Leblanc, N. \& Nattel, S. Cellular mechanisms of atrial contractile dysfunction caused by sustained atrial tachycardia. Circulation. 98, 719-727 (1998).

29. Shin, S. Y. et al. Impaired transport function of the left atrium in patients with lone paroxysmal atrial fibrillation. Echocardiography. 28, 44-51 (2011).

30. Amzulescu, M. S. et al. Myocardial strain imaging: review of general principles, validation, and sources of discrepancies. Eur Heart J Cardiovasc Imaging. 20, 605-619 (2019). 
31. Yoon, Y. E. et al. at al. Echocardiographic Predictors of Progression to Persistent or Permanent Atrial Fibrillation in Patients with Paroxysmal Atrial Fibrillation (E6P Study). J Am Soc Echocardiogr 2015;28:709 - 17.

32. Leung, M. et al. Left atrial function to identify patients with atrial fibrillation at high risk of stroke: new insights from a large registry. Eur Heart J. 39, 1416-1425 (2018).

33. Habibi, M. et al. Association of CMR-measured LA function with heart failure development: results from the MESA study. JACC Cardiovasc Imaging. 7, 570-579 (2014).

34. Hanna, N., Cardin, S., Leung, T. K. \& Nattel, S. Differences in atrial versus ventricular remodeling in dogs with ventricular tachypacing-induced congestive heart failure. Cardiovasc Res. 63, 236-244 (2004).

35. Avitall, B., Bi, J., Mykytsey, A. \& Chicos, A. Atrial and ventricular fibrosis induced by atrial fibrillation: evidence to support early rhythm control. Heart Rhythm. 5, 839-845 (2008).

36. Tsang, T. S. et al. Left ventricular diastolic dysfunction as a predictor of the first diagnosed nonvalvular atrial fibrillation in 840 elderly men and women. J Am Coll Cardiol. 40, 1636-1644 (2002).

37. Rosenberg, M. A. \& Manning, W. J. Diastolic dysfunction and risk of atrial fibrillation: a mechanistic appraisal. Circulation. 126, 2353-2362 (2012).

38. Frustaci, A. et al. Histological substrate of atrial biopsies in patients with lone atrial fibrillation. Circulation. 96, 1180-1184 (1997).

39. Sasaki, N. et al. Transthoracic echocardiographic backscatter-based assessment of left atrial remodeling involving left atrial and ventricular fibrosis in patients with atrial fibrillation. Int $J$ Cardiol. 176, 1064-1066 (2014).

40. Neilan, T. G. et al. Myocardial extracellular volume expansion and the risk of recurrent atrial fibrillation after pulmonary vein isolation. JACC Cardiovasc Imaging. 7, 1-11 (2014).

41. Ellims, A. H. et al. Diffuse myocardial fibrosis evaluated by post-contrast $\mathrm{t} 1$ mapping correlates with left ventricular stiffness. J Am Coll Cardiol. 63, 1112-1118 (2014).

42. Sideris, D. A. et al. Some observations on the mechanism of pressure related atrial fibrillation. Eur Heart J. 15, 1585-1589 (1994).

\section{Figures}




\section{Figure 1}

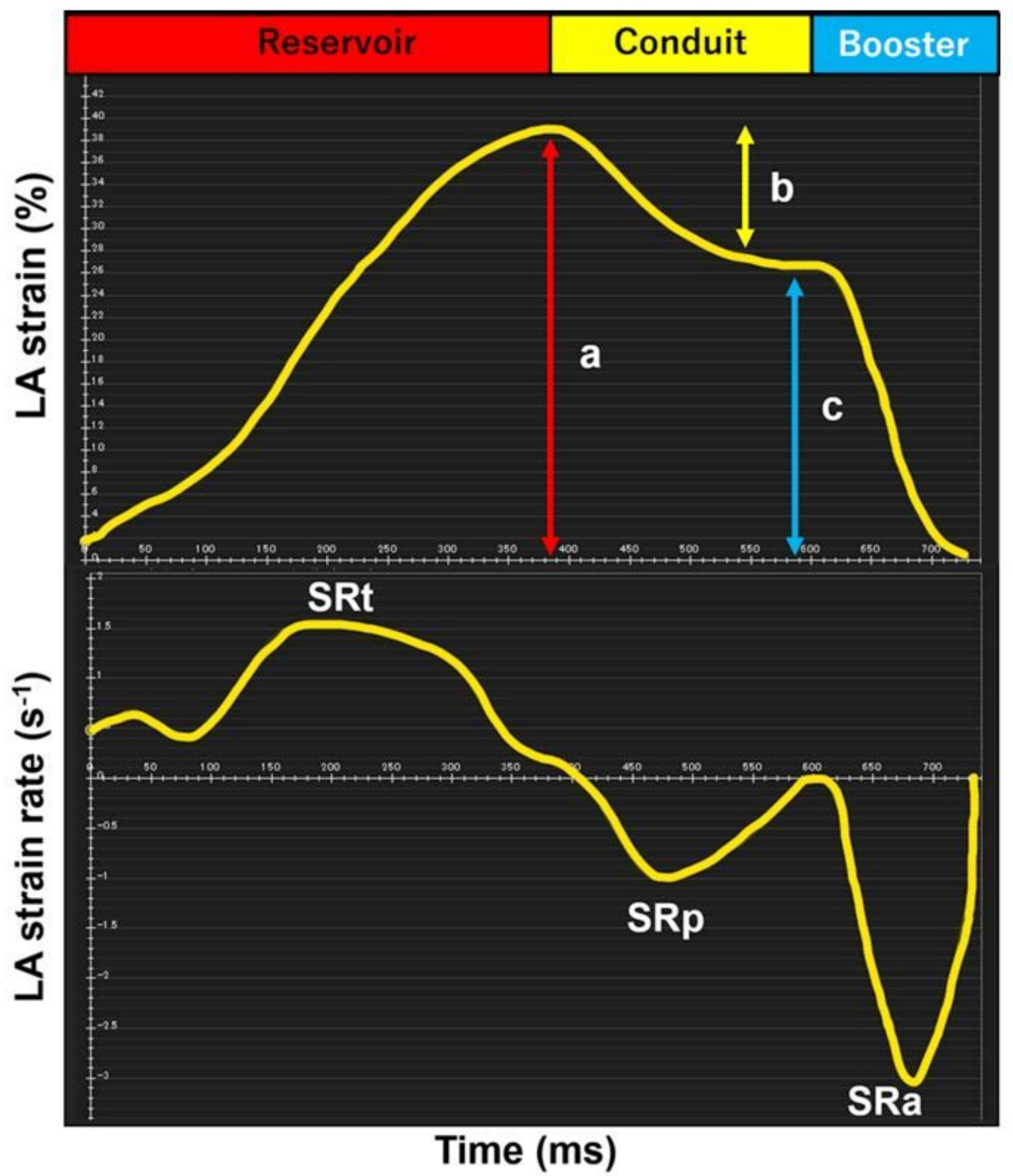

Figure 1

Quantification of left atrial strain and strain rate. Left atrial function comprised three components: reservoir function; conduit function; and booster pump function. Total strain (a) and peak positive strain rate (SRt) correspond to reservoir function. Passive strain (b) and peak early negative strain rate (SRp) correspond to conduit function. Active strain (c) and peak late negative strain rate (SRa) correspond to booster pump function. 


\section{Figure 2}
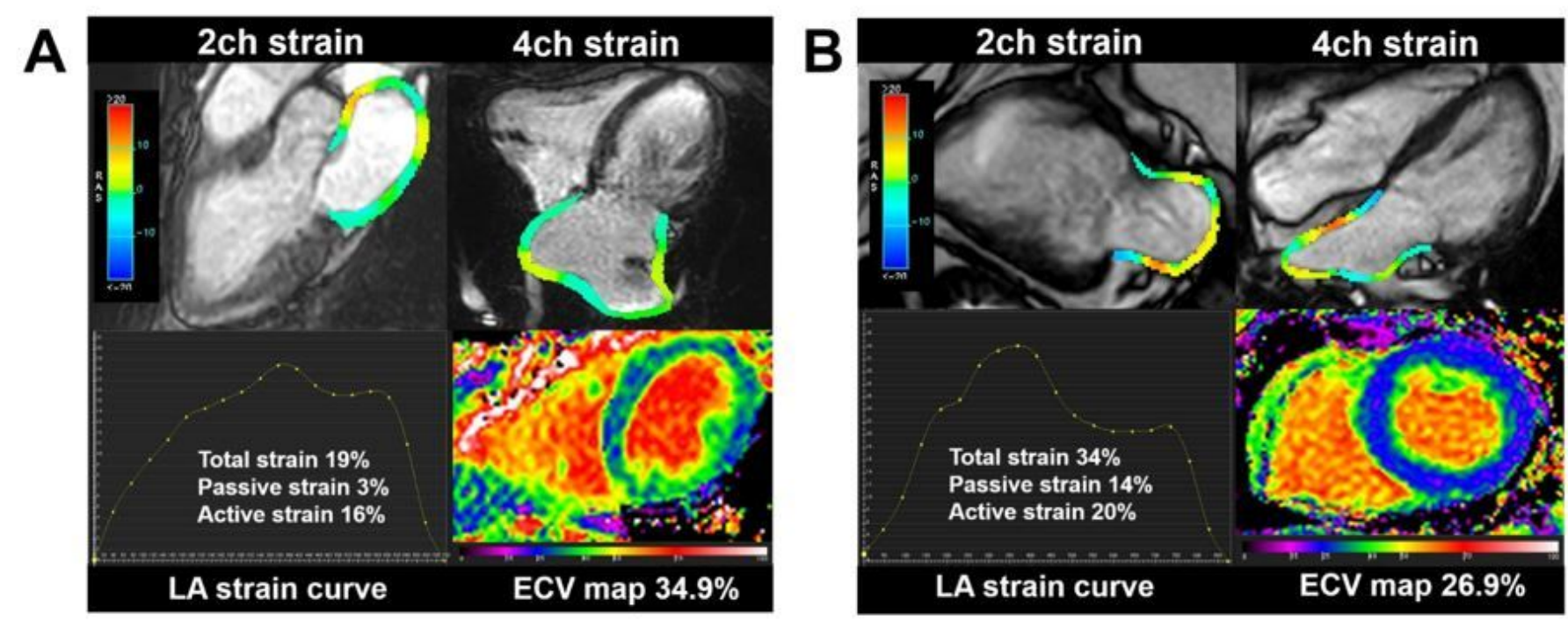

\section{Figure 2}

Representative imaging findings in a patient with PAF (A) and a control subject (B). LV-ECV is higher in the patient with PAF $(34.9 \%)$ than in the control subject (26.9\%), indicating advanced myocardial fibrosis. LA strain analysis reveals that total and passive strain is substantially lower in PAF (total strain: $19 \%$ vs. $34 \%$, passive strain: $3 \%$ vs. $14 \%$ ), while active strain is similar between the patient with PAF and the control subject ( $16 \%$ vs. $20 \%)$. 
Figure 3

\section{ECV (\%)}

32

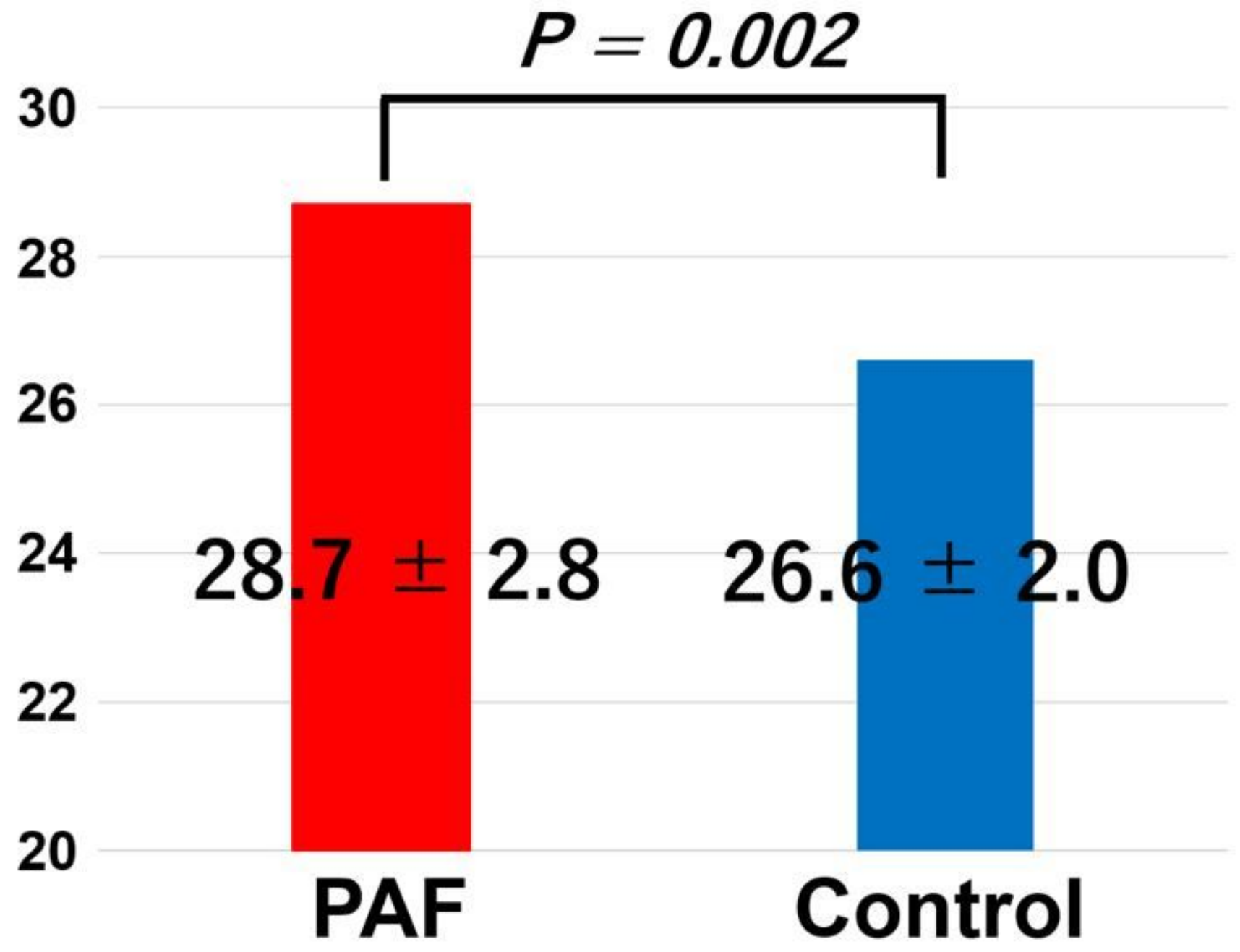

Figure 3

Differences in LV-ECV between the PAF and control groups. LV-ECV is significantly higher in the PAF group $(28.7 \pm 2.8 \%)$ than in the control group $(26.6 \pm 2.0 \%, P=0.002)$.

\section{Supplementary Files}

This is a list of supplementary files associated with this preprint. Click to download.

- Supplementarymaterials02012021.docx 